\title{
On the independence complex of square grids
}

\author{
Mireille Bousquet-Mélou • Svante Linusson • \\ Eran Nevo
}

Received: 8 March 2007 / Accepted: 9 August 2007 / Published online: 12 October 2007

(C) Springer Science+Business Media, LLC 2007

\begin{abstract}
The enumeration of independent sets of regular graphs is of interest in statistical mechanics, as it corresponds to the solution of hard-particle models. In 2004, it was conjectured by Fendley et al., that for some rectangular grids, with toric boundary conditions, the alternating number of independent sets is extremely simple. More precisely, under a coprimality condition on the sides of the rectangle, the number of independent sets of even and odd cardinality always differ by 1 . In physics terms, this means looking at the hard-particle model on these grids at activity -1 . This conjecture was recently proved by Jonsson.
\end{abstract}

Here we produce other families of grid graphs, with open or cylindric boundary conditions, for which similar properties hold without any size restriction: the number of independent sets of even and odd cardinality always differ by $0, \pm 1$, or, in the cylindric case, by some power of 2 .

We show that these results reflect a stronger property of the independence complexes of our graphs. We determine the homotopy type of these complexes using Forman's discrete Morse theory. We find that these complexes are either contractible, or homotopic to a sphere, or, in the cylindric case, to a wedge of spheres.

Finally, we use our enumerative results to determine the spectra of certain transfer matrices describing the hard-particle model on our graphs at activity -1 . These

\footnotetext{
M. Bousquet-Mélou (凶)

CNRS, LaBRI, Université Bordeaux 1, 351 cours de la Libération, 33405 Talence Cedex, France e-mail: mireille.bousquet@labri.fr

S. Linusson

Dept. of Mathematics, KTH-Royal Institute of Technology, 10044 Stockholm, Sweden e-mail: linusson@math.kth.se

E. Nevo

Institute of Mathematics, Hebrew University, Jerusalem, Israel

e-mail: eranevo@math.huji.ac.il
} 
results parallel certain conjectures of Fendley et al., proved by Jonsson in the toric case.

Keywords Hard particles · Independent sets · Independence complex · Discrete Morse theory · Transfer matrices

\section{Introduction}

The hard-square model is a famous open problem in statistical mechanics. In this model, some of the vertices of an $N$ by $N$ square grid are occupied by a particle, with the restriction that two adjacent vertices are never both occupied (Figure 1). In graph theoretic terms, an admissible configuration of particles is just an independent set of the square grid, that is, a set of pairwise non-adjacent vertices. The key question is to enumerate these sets by their size, that is, to determine the following partition function at activity $u$ :

$$
Z_{N}(u)=\sum_{I} u^{|I|},
$$

where the sum runs over all independent sets of the grid. This problem is highly unsolved: one does not know how to express $Z_{N}(u)$, nor even the thermodynamic limit of the sequence $Z_{N}(u)$ (that is, the limit of $Z_{N}(u)^{1 / N^{2}}$ ). The most natural specialization of $Z_{N}(u)$, obtained for $u=1$, counts independent sets of the $N \times N$ grid. It is also extremely mysterious: neither the sequence $Z_{N}(1)$, nor the limit of $Z_{N}(1)^{1 / N^{2}}$ (the so-called hard-square constant) are known. We refer the reader to the entry A006506 in the On-line Encyclopedia of Integer Sequences for more details [11]. Note that the thermodynamic limit of $Z_{N}(u)$ is known if one replaces the square grid by a triangular one — a tour de force achieved by Baxter in 1980 [1].

In 2004, Fendley, Schoutens and van Eerten [3] published a series of remarkable conjectures on the partition function of the hard-square model specialized at $u=$ -1 . For instance, they observed that for an $M \times N$-grid, taken with toric boundary conditions, the partition function at $u=-1$ seemed to be equal to 1 as soon as $M$ and $N$ were coprime. They also related this conjecture to a stronger one, dealing with the eigenvalues of the associated transfer matrices. These conjectures have recently been proved in a sophisticated way by Jonsson [8].

One of the aims of this paper is to prove that similar results hold, in greater generality, for other subgraphs of the square lattice, like the (tilted) rectangles of Figure 2 (they will be defined precisely in Section 3). For these graphs, we prove that the partition function at $u=-1$ is always 0,1 or -1 .

We then show that these results actually reflect a stronger property of the independence complex of these graphs. The independent sets of any graph $G$, ordered by inclusion, form a simplicial complex, denoted by $\Sigma(G)$. See Figure 3, where this complex is shown for a $2 \times 2$-grid $G$. Note that the empty set is an element of $\Sigma(G)$. The (reduced) Euler characteristic of this complex is, by definition:

$$
\tilde{\chi}_{G}=\sum_{I \in \Sigma(G)}(-1)^{|I|-1} .
$$


Fig. 1 A hard particle configuration - or an independent set - of the $7 \times 7$-grid
$(0,0)$

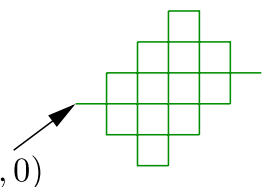

$$
\mathcal{R}(6,8)
$$

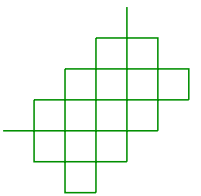

$\mathcal{R}(6,9)$
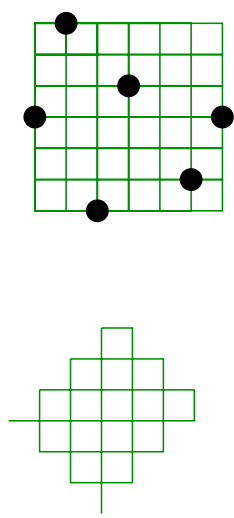

$\mathcal{R}(7,8)$

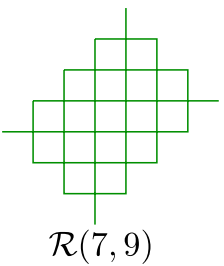

$\mathcal{R}(7,9)$

Fig. 2 The rectangular graphs $\mathcal{R}(M, N)$, defined in Section 3

Fig. 3 Two graphs, and the independence complex of the top one. This complex has reduced Euler characteristic 1, and is homotopic to a 0-dimensional sphere (two points). The patient reader can check that for the "Swiss cross"

$$
\tilde{\mathcal{R}}(3,3) \quad \stackrel{1}{1} \quad{ }_{4}^{2}
$$

$\tilde{\mathcal{R}}(5,5)$

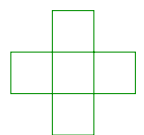

$\{1\}$

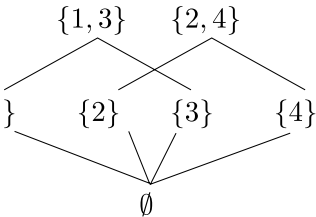

$\tilde{\mathcal{R}}(5,5)$, the reduced Euler

characteristic is -1 . The

corresponding complex is

homotopic to a 3-dimensional

sphere

The quantity $|I|-1$ is the dimension of the cell $I$. The above sum is exactly the opposite of the partition function $Z_{G}(u)$ of the hard-particle model on $G$, evaluated at $u=-1$. This number,

$$
Z_{G}(-1)=\sum_{I \in \Sigma(G)}(-1)^{|I|}=-\tilde{\chi}_{G}
$$

will often be called the alternating number of independent sets. The simplicity of the Euler characteristic for certain graphs $G$ suggests that the complex $\Sigma(G)$ could have a very simple homotopy type (we refer to Munkres [10] for the topological terms involved). We prove that this is indeed the case for various subgraphs of the square lattice. For instance, for the rectangles of Figure 2, the independence complex is always either contractible, or homotopy equivalent to a sphere. Our results rely on the construction of certain Morse matchings of the complex $\Sigma(G)$. Roughly speaking, these matchings are parity reversing involutions on $\Sigma(G)$ having certain additional interesting properties.

Let us now describe the contents of this paper, and compare it to Jonsson's results. In Section 2, we first review the needed background of Forman's discrete Morse theory [4]. We then describe a general construction of Morse matchings for the indepen- 
dence complex of any graph. The matchings we construct are encoded by a matching tree. In Sections 3 to 5, we apply this general machinery to determine the homotopy type of the independence complex of several subgraphs of the square grid: the tilted rectangles of Figures 2 and 5 (Section 3), the parallelograms of Figure 9 (Section 5), and variations on them (Section 7.1). All these graphs have open boundary conditions (as opposed to the toric boundary conditions of $[3,8]$ ). However, in Section 4, we identify two sides of the rectangles of Figure 2 to obtain rectangles with cylindric boundary conditions. Again, we determine the homotopy type of the associated independence complex. Note that Jonsson recently went one step further by studying the same tilted rectangles with toric boundary conditions; but he was only able to determine the Euler characteristic ([7], Section 7). Our results deal with a finer invariant of the complex (the homotopy type) and the proofs are simpler, but they also refer to easier graphs; the toric case is at the moment beyond reach of our methods.

Finally, in Section 6 we give background about transfer matrices, and show how the results of the previous sections can be used to derive the spectrum, or at least part of the spectrum, of several transfer matrices naturally associated to our graphs.

We conclude the paper with a discussion on possible extensions of our work, with the double objective of discovering new subgraphs with a simple alternating number of independent sets, and addressing the conjectures of Fendley et al. in the cylindric case.

\section{Morse matchings for independence complexes}

The key tool in the proof of our results is the construction of Morse matchings on independence complexes. In this section we give the background and explain how we use a matching tree as a systematic construction for Morse matchings.

\subsection{Generalities}

Let us first recall that the poset of independent sets of a graph $G$, ordered by inclusion, is a simplicial complex, denoted $\Sigma(G)$.

We regard any finite poset $P$ as a directed graph, by considering the Hasse diagram of $P$ with edges pointing down (that is, from larger to smaller elements).

A set $M$ of pairwise disjoint edges of this graph is called a matching of $P$. This matching is perfect if it covers all elements of $P$. This matching is Morse (or acyclic) if the directed graph obtained from $P$ by reversing the direction of the edges in $M$ is acyclic. For instance, the matching of the complex of Figure 3 formed of the edges $(\emptyset,\{2\}),(\{3\},\{1,3\}),(\{4\},\{2,4\})$ is acyclic.

Theorem 1 ([5], Theorem 6.3) Let $\Sigma$ be a finite simplicial complex, seen as a poset, and $M$ a Morse matching on $\Sigma$, such that the element $\emptyset$ of $\Sigma$ is matched. For $i \geq 0$, let $n_{i}$ be the number of unmatched $i$-dimensional elements of $\Sigma$. Then there exists a $C W$-complex having $1+n_{0} 0$-dimensional cells and $n_{i} i$-dimensional cells for $i \geq 1$ that is homotopy equivalent to $\Sigma$. 
Again, we refer to [10] for the topological terms involved. We will only use the following immediate corollary.

Corollary 2 Under the assumptions of Theorem 1, if all unmatched elements in $\Sigma$ have the same dimension $i>0$ and there are $j$ of them, $\Sigma$ is homotopy equivalent to a wedge of $j$ spheres of dimension $i$. In particular, if $\Sigma$ is perfectly matched, then it is contractible.

\subsection{Matching trees}

Let us now describe the general principle we use to construct Morse matchings for a complex $\Sigma(G)$. Let $V$ denote the vertex set of $G$. The most naive way to define a matching of $\Sigma \equiv \Sigma(G)$ is probably the following. Take a vertex $p \in V$, and denote by $N(p)$ the set of its neighbors. Define

$$
\Delta=\{I \in \Sigma: I \cap N(p)=\emptyset\} .
$$

The set of pairs $(I, I \cup\{p\})$, for $I \in \Delta$ and $p \notin I$, forms a perfect matching of $\Delta$, and hence a matching of $\Sigma$. We call $p$ the pivot of this matching. The unmatched elements of $\Sigma$ are those containing at least one element of $N(p)$. There may be many unmatched elements, but we can now choose another pivot $p^{\prime}$ to match some elements of $\Sigma \backslash \Delta$, and repeat this operation as long as we can. Of course, the resulting matching will depend on the successive choices of pivots.

This rather naive idea is the leading thread in the construction of our Morse matchings of $\Sigma$. In some occasions, we will have to split the set of yet unmatched elements into two subsets, and choose a different pivot for each of them. This explains why our matching procedure will be encoded by a branching structure, namely a plane rooted tree, called a matching tree of $\Sigma$. The nodes of this tree represent sets of yet unmatched elements. Some nodes are reduced to the empty set, and all the others are subsets of $\Sigma$ of the form

$$
\Sigma(A, B)=\{I \in \Sigma: A \subseteq I \text { and } B \cap I=\emptyset\}
$$

where $A$ and $B$ are two subsets of $V$ such that

$$
A \cap B=\emptyset \quad \text { and } \quad N(A):=\cup_{a \in A} N(a) \subseteq B .
$$

We say that the vertices of $A \cup B$ are the prescribed vertices of $\Sigma(A, B)$ : any element $I$ of $\Sigma(A, B)$ must contain the vertices of $A$ and avoid those of $B$. In particular, it is assumed that $A$ itself is independent (otherwise $\Sigma(A, B)$ would be empty). The root of the tree is $\Sigma(\emptyset, \emptyset)=\Sigma$, the set of all independent sets of $G$. The sets $A$ and $B$ will increase along branches, making the sets $\Sigma(A, B)$ of unmatched elements smaller and smaller. The leaves of the tree will have cardinality 0 or 1 , and will contain the elements that are left unmatched at the end of the procedure.

Consider a node of the tree of the form $\Sigma(A, B)$. How can we match its elements? If the node has cardinality 1 , that is, if $A \cup B=V$, then we are stuck, as there is no non-trivial matching of a graph reduced to one vertex. If $A \cup B \subsetneq V$, we may match 
some of the elements of $\Sigma(A, B)$. Pick a vertex $p$ in $V^{\prime}:=V \backslash(A \cup B)$. Because of (2), the neighbors of $p$ are either in $B$, or in $V^{\prime}$. This makes $p$ a good tentative pivot. If we actually use $p$ as a pivot to match elements of $\Sigma(A, B)$, we will be left with the following set of unmatched elements:

$$
U=\{I \in \Sigma: A \subseteq I, I \cap B=\emptyset, I \cap N(p) \neq \emptyset\} .
$$

If $p$ has no neighbor in $V^{\prime}$, the above set is empty, and we have perfectly matched $\Sigma(A, B)$. If $p$ has exactly one neighbor in $V^{\prime}$, say $v$, then $U=\Sigma(A \cup\{v\}, B \cup N(v))$. However, if $p$ has at least two neighbors in $V^{\prime}$, say $v$ and $v^{\prime}$, the set $U$ is not of the form $\Sigma\left(A^{\prime}, B^{\prime}\right)$. Indeed, some of the unmatched sets $I$ contain $v$, some others don't, but then they have to contain $v^{\prime}$. This puts us into trouble, as we want to handle only unmatched sets of the form $\Sigma\left(A^{\prime}, B^{\prime}\right)$. We circumvent this difficulty by splitting the original set $\Sigma(A, B)$ into two disjoint subsets of the form $\Sigma\left(A^{\prime}, B^{\prime}\right)$, that differ by the status of, say, the vertex $v$. More precisely, we write:

$$
\Sigma(A, B)=\Sigma(A, B \cup\{v\}) \uplus \Sigma(A \cup\{v\}, B \cup N(v)),
$$

and then study separately each subset.

The above discussion justifies the following construction of the children of a node. If this node is the empty set (no unmatched elements), we declare it a leaf. Otherwise, the node is of the form $\Sigma(A, B)$. If $A \cup B=V$, then $\Sigma(A, B)=\{A\}$ is a node of cardinality 1 , and we also declare it a leaf. We are left with nodes of the form $\Sigma(A, B)$, with $A \cup B \subsetneq V$. Choose a vertex $p$ (the tentative pivot) in $V^{\prime}=V \backslash(A \cup$ $B)$, and proceed as follows:

- If $p$ has at most one neighbor in $V^{\prime}$, define $\Delta(A, B, p)$ to be the subset of $\Sigma(A, B)$ formed of sets that do not intersect $N(p)$ :

$$
\Delta(A, B, p)=\{I \in \Sigma: A \subseteq I \text { and } B \cap I=I \cap N(p)=\emptyset\}
$$

Let $M(A, B, p)$ be the perfect matching of $\Delta(A, B, p)$ obtained by using $p$ as a pivot. Give to the node $\Sigma(A, B)$ a unique child, namely the set $U=\Sigma(A, B) \backslash$ $\Delta(A, B, p)$ of unmatched elements. This set is empty if $p$ has no neighbor in $V^{\prime}$. In this case, we say that $p$ is a free vertex of $\Sigma(A, B)$. If $p$ has exactly one neighbor $v$ in $V^{\prime}$, then $U=\Sigma(A \cup\{v\}, B \cup N(v))$. Index the new edge by the pivot $p$. We say that the 3-tuple $(A, B, p)$ is a matching site of the tree.

- Otherwise, let us choose one neighbor $v$ of $p$ in $V^{\prime}$. The node $\Sigma(A, B)$ has two children, only differing by the status of $v$. More precisely, the left child is $\Sigma(A, B \cup\{v\})$ and the right child is $\Sigma(A \cup\{v\}, B \cup N(v))$. The union of these two sets is $\Sigma(A, B)$. Label the two new edges by the splitting vertex $v$. We say that $(A, B, v)$ is a splitting site of the tree.

Observe that the new nodes satisfy Conditions (2), unless they are empty.

Given a sequence of choices of tentative pivots and splitting vertices, we obtain a matching $M$ of $\Sigma$ by taking the union of all partial matchings $M(A, B, p)$ performed at the matching sites of the tree. The unmatched elements are those sitting at the leaves of the tree. 


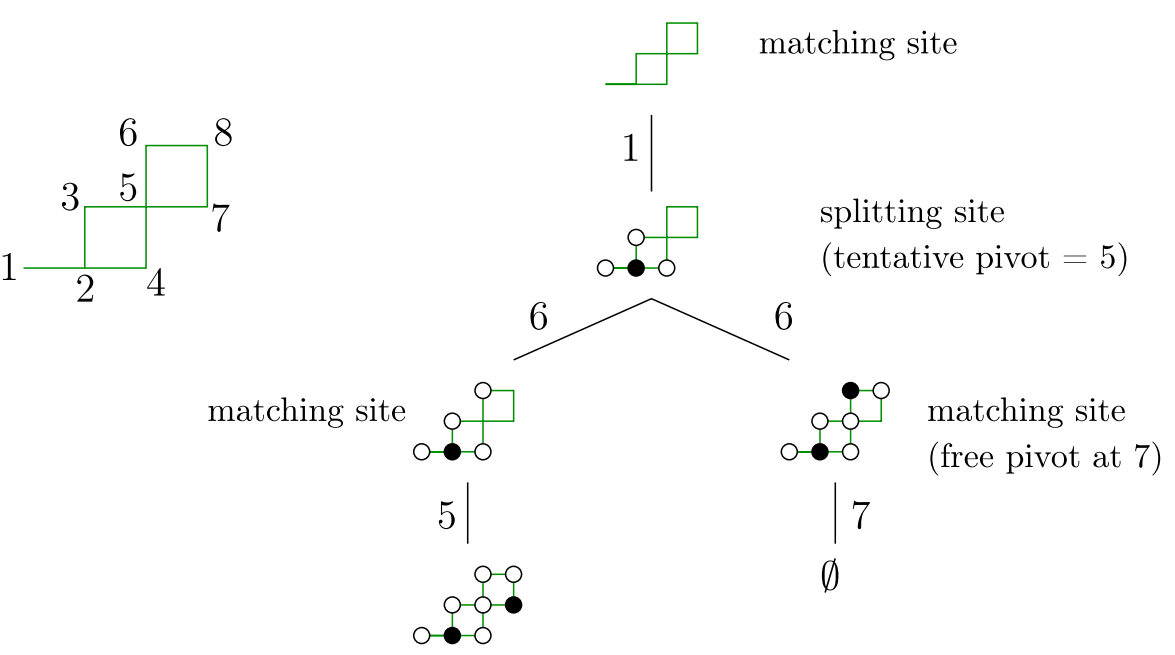

Fig. 4 A subgraph $G$ of the square grid, and one of its matching trees

The above construction is rather natural, and we invite the reader to practice with the example given in Figure 4. In this figure, every (non-empty) node is described by the vertices of $A$ (in black) and $B$ (in white). At the matching site $(\emptyset, \varnothing, 1)$, the elements $\emptyset$ and $\{1\}$ (among others) are matched. At the matching site $(\{2\},\{1,3,4,6\}, 5)$, the elements $\{2\}$ and $\{2,5\}$ get matched, among others. At the end of the matching procedure, the independent set $\{2,7\}$ is the only unmatched element of $\Sigma(G)$.

We now aim at showing that the matchings obtained with the above procedure are in fact Morse. The following lemma gathers some properties of this construction.

Lemma 3 Every matching tree satisfies the following properties:

(1) For every matching site $(A, B, p)$, the matching $M(A, B, p)$ is a Morse matching of $\Delta(A, B, p)$ (still ordered by inclusion).

(2) Let $(A, B, p)$ be a matching site with a non-empty child $\Sigma(A \cup\{v\}, B \cup N(v))$. Let $I \in \Delta(A, B, p)$ and $J \in \Sigma(A \cup\{v\}, B \cup N(v))$. Then $J \nsubseteq I$.

(3) Let $(A, B, v)$ be a splitting site, $I \in \Sigma(A, B \cup\{v\})$ and $J \in \Sigma(A \cup\{v\}, B \cup$ $N(v))$. Then $J \nsubseteq I$.

Proof (1) Consider the Hasse diagram of the poset $\Delta(A, B, p)$ and its directed version, with all edges pointing down. Now, reverse the edges of $M(A, B, p)$. The up edges join two elements of the form $I \backslash\{p\}, I$, so they correspond to adding the vertex $p$. The down edges correspond to deleting a vertex different from $p$. Clearly there cannot be a directed cycle in $\Delta(A, B, p)$.

(2) The set $J$ contains $v$, a neighbor of the pivot $p$, while none of the matched sets $I$ of $\Delta(A, B, p)$ contains $v$.

(3) Here again, $J$ contains $v$, but $I$ doesn't.

The following easy lemma appears as Lemma 4.3 in Jonsson's thesis [6]. 
Lemma 4 Let $V$ be a finite set and $\Delta=\Delta_{1} \uplus \Delta_{2}$ a collection of subsets of $V$, ordered by inclusion. Assume that if $\sigma \in \Delta_{1}$ and $\tau \in \Delta_{2}$ then $\tau \nsubseteq \sigma$. Then the union of two acyclic matchings on $\Delta_{1}$ and $\Delta_{2}$ respectively is an acyclic matching on $\Delta$.

We can now establish the main result of this section.

Proposition 5 For any graph $G$ and any matching tree of $G$, the matching of $\Sigma(G)$ obtained by taking the union of all partial matchings $M(A, B, p)$ performed at the matching sites is Morse.

Proof We will prove by backward induction, from the leaves to the root, the following property:

For every node $\tau$ of the matching tree, the union of the partial matchings performed at the descendants of $\tau$ (including $\tau$ itself) is a Morse matching of $\tau$. We denote this matching $U M(\tau)$ (for Union of Matchings).

The leaves of the tree are either empty sets or singletons, endowed with the empty matching, which is Morse. This supplies the induction base. Consider now a non-leaf node of the tree, of the form $\tau=\Sigma(A, B)$.

Assume $(A, B, p)$ is a matching site. By Lemma 3.1, $M(A, B, p)$ is Morse. If the (unique) child of $\tau$ is empty, then $U M(\tau)=M(A, B, p)$ and we are done. If this child is $\tau^{\prime}=\Sigma(A \cup\{v\}, B \cup N(v))$, the induction hypothesis tells us that $U M\left(\tau^{\prime}\right)$ is Morse. By Lemma 3.2, we can apply Lemma 4 with $\Delta_{1}=\Delta(A, B, p), \Delta_{2}=\tau^{\prime}$ and $\Delta=\tau$, where the partial matchings on $\Delta_{1}$ and $\Delta_{2}$ are respectively $M(A, B, p)$ and $U M\left(\tau^{\prime}\right)$. This shows that $U M(\tau)$ is Morse.

Assume $(A, B, v)$ is a splitting site. By induction hypothesis we already have Morse matchings on both children of $\tau$, namely $\Delta_{1}=\Sigma(A, B \cup\{v\})$ and $\Delta_{2}=$ $\Sigma(A \cup\{v\}, B \cup N(v))$. Again, Lemma 3.3 allows us to apply Lemma 4, and this shows that the union $U M(\tau)$ of $U M\left(\Delta_{1}\right)$ and $U M\left(\Delta_{2}\right)$ is Morse.

This completes the induction. The case where $\tau$ is the root of the tree gives the proposition.

\section{Rectangles with open boundary conditions}

In what follows, we consider $\mathbf{Z}^{2}$ as an infinite graph, with edges joining vertices at distance 1 from each other. For $M, N \geq 1$, let $\mathcal{R}(M, N)$ be the subgraph of $\mathbf{Z}^{2}$ induced by the points $(x, y)$ satisfying

$$
y \leq x \leq y+M-1 \text { and } \quad-y \leq x \leq-y+N-1
$$

Examples are shown on Figure 2. Note that $\mathcal{R}(M, N)$ contains $\left\lceil\frac{M N}{2}\right\rceil$ vertices. Other rectangular shapes arise when we look at the subgraph $\tilde{\mathcal{R}}(M, N)$ of $\mathbf{Z}^{2}$ induced by the points $(x, y)$ such that

$$
y \leq x \leq y+M-1 \quad \text { and } \quad-y+1 \leq x \leq-y+N
$$




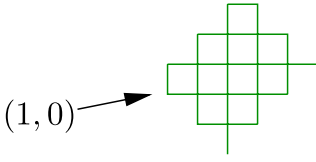

$\tilde{\mathcal{R}}(6,7)$

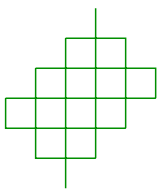

$\tilde{\mathcal{R}}(6,8)$

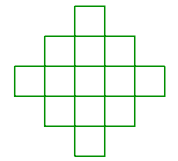

$\tilde{\mathcal{R}}(7,7)$

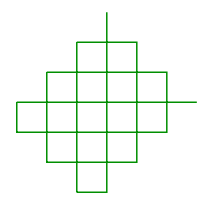

$\tilde{\mathcal{R}}(7,8)$

Fig. 5 The graphs $\tilde{\mathcal{R}}(M, N)$

(see Figure 5). More precisely, the graphs $\tilde{\mathcal{R}}(2 M+1,2 N+1)$ are not isomorphic to any of the $\mathcal{R}(K, L)$ (the other graphs $\tilde{\mathcal{R}}(M, N)$ are isomorphic to an $\mathcal{R}(K, L))$.

We study the independence complexes of the rectangles $\mathcal{R}(M, N)$ and $\tilde{\mathcal{R}}(M, N)$. Recall the general connection (1) between the (reduced) Euler characteristic of these complexes and the alternating number of independent sets. We use below the notation $Z_{\mathcal{R}}(M, N)$ rather than $Z_{\mathcal{R}(M, N)}$.

Theorem 6 Let $M, N \geq 1$. Let $m=\lceil M / 3\rceil$ and $n=\lceil N / 3\rceil$.

- If $M \equiv_{3} 1$ or $N \equiv_{3} 1$, then $\Sigma(\mathcal{R}(M, N))$ is contractible and $Z_{\mathcal{R}}(M, N)=0$.

- Otherwise, $\Sigma(\mathcal{R}(M, N))$ is homotopy equivalent to a sphere of dimension mn - 1 , and $Z_{\mathcal{R}}(M, N)=(-1)^{m n}$.

The above holds also when replacing $\mathcal{R}(M, N)$ by $\tilde{\mathcal{R}}(M, N)$.

\section{Remark}

1. We will show in Corollary 10 that, for $N \equiv{ }_{3} 1$ and $M>2^{1+\lceil N / 2\rceil}$, the alternating number $Z_{\mathcal{R}}(M, N ; C, D)$ of independent sets of $\mathcal{R}(M, N)$ having border conditions $C$ and $D$ on the two extreme diagonals of slope 1 is actually 0 for all configurations $C$ and $D$. This will indirectly follow from the study of tilted rectangles with cylindric boundary conditions performed in Section 4.

2. The case $N=2$, where the graph is a path of $M$ vertices, has already been solved in [9, Prop. 4.6].

Proof We study the graphs $\mathcal{R}(M, N)$ and $\tilde{\mathcal{R}}(M, N)$ together. We construct Morse matchings of the independence complexes of these graphs by following the general principles of Section 2. We need to specify our choice of tentative pivots and splitting vertices. The objective is to minimize the combinatorial explosion of cases, that is, the number of splitting sites. Our conventions are illustrated by an example in Figure 6. As before, for any node $\Sigma(A, B)$, the elements of $A$ and $B$ are indicated by $\bullet$ and $\circ$ respectively. Rather than labeling the vertices of the graph and the edges of the tree, we have indicated the tentative pivots by $*$, and the splitting vertices by $\triangle$. Consider a node $\Sigma(A, B)$.

(1) In general, the tentative pivot $p=\left(i_{0}, j_{0}\right)$ is chosen in $V^{\prime}=V \backslash(A \cup B)$ so as to minimize the pair $(i+j, i)$ for the lexicographic order. That is, $p$ lies as high as possible on the leftmost diagonal of slope -1 . However, if there is at least one 
Fig. 6 The top of the tree describing the matching of $\Sigma(\mathcal{R}(8,6))$

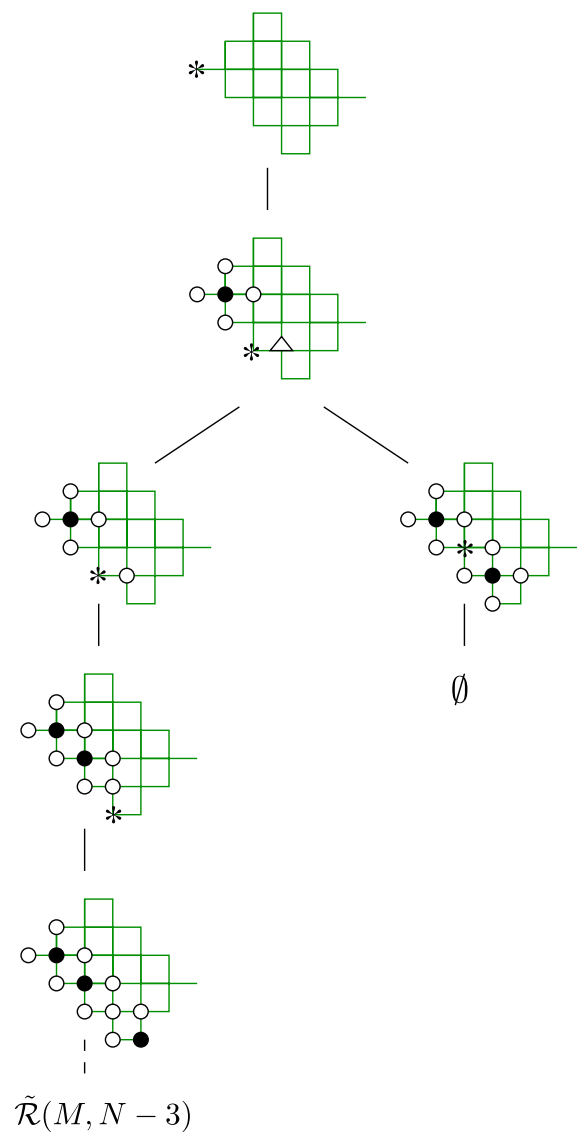

free vertex (that is, a vertex of $V^{\prime}=V \backslash(A \cup B)$ having no neighbor in $\left.V^{\prime}\right)$ on the next diagonal $i+j=i_{0}+j_{0}+1$, then we choose one of them as the pivot: the only child of $\Sigma(A, B)$ is then the empty set.

(2) If the tentative pivot has several neighbors in $V^{\prime}=V \backslash(A \cup B)$, then it has exactly two neighbors in $V^{\prime}$, namely its North and East neighbors. Indeed, the other two neighbors come before $p$ in the lexicographic order, and thus belong to $A \cup B$. Take $v$, the splitting vertex, to be the East neighbor of $p$.

We are going to prove by induction on $N$ and $M$ the following properties, valid both for the graphs $\mathcal{R}(M, N)$ and $\tilde{\mathcal{R}}(M, N)$ :

(A) if $M \equiv_{3} 1$ or $N \equiv_{3} 1$, then there is no unmatched element in $\Sigma$,

$(B)$ otherwise, there is a unique unmatched element, of cardinality $m n$ (and thus of dimension $m n-1)$.

By Proposition 5, the matchings we obtain are Morse. Hence Theorem 6 follows from Properties $(A)$ and $(B)$ using Corollary 2. Let us now prove these properties. We first study small values of $N$. 
(1) If $N=1$, the graph is formed of isolated points, so that any set of vertices is independent. The matching $M(\emptyset, \emptyset, p)$ performed at the root of the tree is a perfect matching. Property $(A)$ follows.

(2) For $N=2$, we leave it to the reader to check $(A)$ and (B) for $M=1,2,3$, both for the graphs $\mathcal{R}(M, N)$ and $\tilde{\mathcal{R}}(M, N)$. We then proceed by induction on $M$, for $M \geq 4$.

(a) For the graph $\mathcal{R}(M, 2)$, the root is a matching site, and its unique child is $\Sigma(A, B)$ with $A=\{(1,0)\}$ and $B=\{(0,0),(1,-1)\}$. The graph obtained by deleting the vertices of $A \cup B$ is (a translate of) $\tilde{\mathcal{R}}(M-3,2)$. This shows that every unmatched element of $\Sigma(\mathcal{R}(M, 2))$ is obtained by adding the vertex $(1,0)$ to (a translate of $)$ an unmatched element of $\Sigma(\tilde{\mathcal{R}}(M-3,2))$. The result then follows by induction on $M$.

(b) For the graph $\tilde{\mathcal{R}}(M, 2)$, the root is a splitting site. Its right child is perfectly matched using the free pivot $(1,1)$, and has the empty set as its unique child. The left child of the root is partially matched using the pivot $(1,0)$. The unmatched elements are those of $\Sigma(A, B)$, with $A=\{(1,1)\}$ and $B=\{(1,0),(2,0)\}$. The graph obtained by deleting the vertices of $A \cup B$ is (a translate of) $\mathcal{R}(M-3,2)$. This shows that every unmatched element of $\Sigma(\tilde{\mathcal{R}}(M, 2))$ is obtained by adding the vertex $(1,1)$ to (a translate of) an unmatched element of $\Sigma(\mathcal{R}(M-3,2))$. The result then follows by induction on $M$.

The study of the case $N=2$ leads us to introduce a notation that will be useful in our forthcoming inductions.

Notation 1 Let $V$ be a subset of vertices of the square grid, and assume that there exist $i, j \in \mathbb{Z}$ such that $V$ is the disjoint union $V=V_{1} \uplus\left(V_{2}+(i, j)\right.$ ) (where $V_{2}+$ $\left.(i, j)=\left\{v+(i, j): v \in V_{2}\right\}\right)$. Let $X_{1}$ and $X_{2}$ be two collections of sets on the ground sets $V_{1}$ and $V_{2}$, respectively. Then $Y:=\left\{I_{1} \uplus\left(I_{2}+(i, j)\right): I_{1} \in X_{1}, I_{2} \in X_{2}\right\}$ is a collection of sets on the ground set $V$. We use the notation $Y \cong X_{1} * X_{2}$ to denote that the elements of $Y$ are formed by the concatenation of an element of $X_{1}$ with (the translate of) an element of $X_{2}$. In particular, $|Y|=\left|X_{1}\right|\left|X_{2}\right|$.

For instance, if $U(M, N)$ (resp. $\tilde{U}(M, N))$ denotes the set of unmatched elements in $\Sigma(\mathcal{R}(M, N))$ (resp. $\Sigma(\tilde{\mathcal{R}}(M, N)))$, the above observations can be summarized by

$$
U(M, 2) \cong U(3,2) * \tilde{U}(M-3,2) \text { and } \tilde{U}(M, 2) \cong \tilde{U}(3,2) * U(M-3,2) .
$$

We now return to our induction.

(3) The case $N=3$ is very similar to the case $N=2$. One first checks that the result holds for $M=1,2,3$, both for the graphs $\mathcal{R}(M, N)$ and $\tilde{\mathcal{R}}(M, N)$. For $M \geq 4$, the result is proved by induction on $M$, after observing that

$$
U(M, 3) \cong U(3,3) * \tilde{U}(M-3,3) \text { and } \quad \tilde{U}(M, 3) \cong \tilde{U}(3,3) * U(M-3,3)
$$

The following three observations will be useful in the rest of the proof. Firstly, the tentative pivot is never taken in the third diagonal of slope -1 . Secondly, when it 
is taken in the second diagonal, it is a free pivot. Finally, when the matching tree has a non-empty leaf $\Sigma(A, B)$ (that is, when $\left.M \not \equiv_{3} 1\right)$, all vertices on the third diagonal belong to $B$.

(4) Now, let $N \geq 4$. The key observation is that the top of the matching tree coincides, as far as the prescribed vertices, pivots and splitting sites are concerned, with the matching tree obtained for $N=3$. This is illustrated in Figure 6, and holds as long as the pivots are taken in the first two diagonals. Once these pivots have been exhausted, we are left with (at most) one non-empty unmatched set $\Sigma(A, B)$, whose prescribed vertices are those of $U(M, 3)$ (if we work with $\mathcal{R}(M, N)$ ), or $\tilde{U}(M, 3)$ (if we work with $\tilde{\mathcal{R}}(M, N))$. Moreover, the tree rooted at the vertex $\Sigma(A, B)$ is isomorphic to the matching tree of $\tilde{\mathcal{R}}(M, N-3)$ (resp. $\mathcal{R}(M, N-3))$. This leads to

$$
\begin{aligned}
& U(M, N) \cong U(M, 3) * \tilde{U}(M, N-3), \\
& \tilde{U}(M, N) \cong \tilde{U}(M, 3) * U(M, N-3)
\end{aligned}
$$

when for $M \equiv_{3} 1$ both $U(M, 3)$ and $\tilde{U}(M, 3)$ are empty. Properties $(A)$ and $(B)$ easily follow.

\section{Rectangles with cylindric boundary conditions}

We now study a "cylindric" version of the graphs $\mathcal{R}(M, N)$, obtained by wrapping these graphs on a cylinder. For $M, N \geq 0$ and $M$ even, we consider the graph $\mathcal{R}^{c}(M, N)$ obtained from $\mathcal{R}(M+1, N)$ by identifying the vertices $(i, i)$ and $(M / 2+i,-M / 2+i)$, for $0 \leq i \leq\left\lfloor\frac{N-1}{2}\right\rfloor$. Observe that the rectangles $\tilde{\mathcal{R}}(M, N)$ of Figure 5, when wrapped in a natural way around a cylinder, yield the same family of graphs. We denote by $Z_{\mathcal{R}}^{c}(M, N)$ the alternating number of independent sets on the graph $\mathcal{R}^{c}(M, N)$.

Theorem 7 Let $M, N \geq 1$, with $M$ even. Let $m=\left\lfloor\frac{M+1}{3}\right\rfloor$ and $n=\lceil N / 3\rceil$.

- If $N \equiv_{3} 1$, then $\Sigma\left(\mathcal{R}^{c}(M, N)\right)$ is contractible and $Z_{\mathcal{R}}^{c}(M, N)=0$.

- Otherwise,

- If $M \equiv_{3} 0$, then $\Sigma\left(\mathcal{R}^{c}(M, N)\right)$ is homotopy equivalent to a wedge of $2^{n}$ spheres of dimension $m n-1$, and $Z_{\mathcal{R}}^{c}(M, N)=2^{n}$.

- If $M \equiv_{3} 1$ or 2 then $\Sigma\left(\mathcal{R}^{c}(M, N)\right)$ is homotopy equivalent to a single sphere of dimension $m n-1$, and $Z_{\mathcal{R}}^{c}(M, N)=(-1)^{n}$.

The case $N=2$, where the graph is a ring of $M$ vertices, has already been solved in [9, Prop. 5.2].

Proof We define a matching of $\Sigma \equiv \Sigma\left(\mathcal{R}^{c}(M, N)\right)$ by adopting the same choice of tentative pivots and splitting vertices as in the proof of Theorem 6 .

We are going to prove by induction on $N$ the following properties: 
Fig. 7 The top of the matching tree of $\Sigma\left(\mathcal{R}^{c}(8,3)\right)$

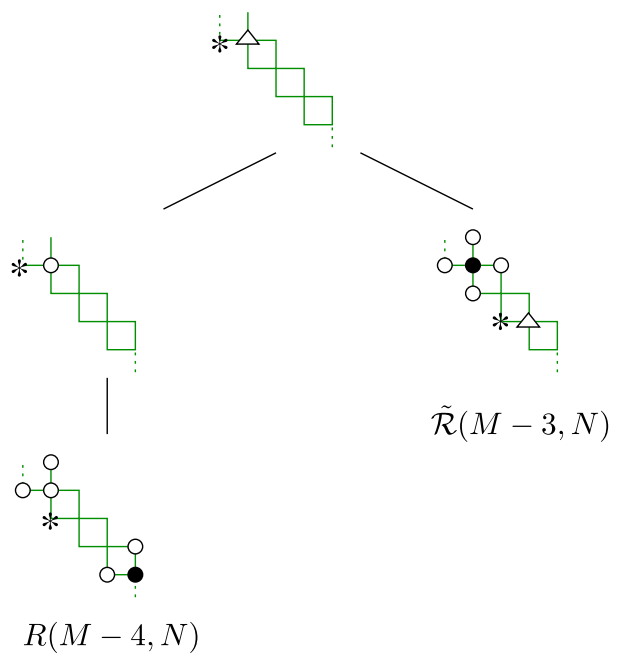

(A) if $N \equiv_{3} 1$, then there is no unmatched element in $\Sigma$,

(B) otherwise,

$\left(B_{1}\right)$ If $M \equiv_{3} 0$, there are $2^{n}$ unmatched elements, each of cardinality $m n$.

$\left(B_{2}\right)$ If $M \equiv_{3} 1$ or 2 there is a unique unmatched element, of cardinality $m n$.

The theorem then follows from Proposition 5 and Corollary 2. Properties $(A)$ and (B) are proved by induction on $N$.

(1) When $N=1$, the graph is formed of isolated vertices, and the first pivot already matches $\Sigma\left(\mathcal{R}^{c}(M, N)\right)$ perfectly.

(2) When $N=2$ or $N=3$, we begin the matching procedure, and then use the results obtained in the previous section for rectangles with open boundary conditions. The root of the matching tree is a splitting site (Figure 7). Its right subtree is isomorphic to the matching tree of $\tilde{\mathcal{R}}(M-3, N)$. Its left subtree begins with a matching site. Performing this partial matching leads to a new node, and the tree starting at this node is isomorphic to the matching tree of $\mathcal{R}(M-4, N)$. Theorem 6 then implies that the matching tree of $\mathcal{R}^{c}(M, N)$ has two non-empty leaves corresponding to unmatched cells of cardinality $m=M / 3$ if $M \equiv_{3} 0$, and one non-empty leaf otherwise, of cardinality $m$. The full matching tree can be seen on Figure 8 (retain only the first two or three diagonals). If $N=3$, we observe that for each non-empty leaf $\Sigma(A, B)$, all vertices on the rightmost diagonal belong to $B$. This was also true for rectangles with open boundary conditions, and is crucial for the rest of the induction on $N$.

(3) For $N \geq 4$, the top of the tree coincides again with the matching tree of $\mathcal{R}^{c}(M, 3)$. Once the pivots of the first two diagonals have been exhausted, the prescribed vertices are exactly those of the first three diagonals (Figure 8). Denoting by $U^{c}(M, N)$ the set of unmatched elements of $\Sigma\left(\mathcal{R}^{c}(M, N)\right)$, it follows that:

$$
U^{c}(M, N) \cong U^{c}(M, 3) * U^{c}(M, N-3) .
$$



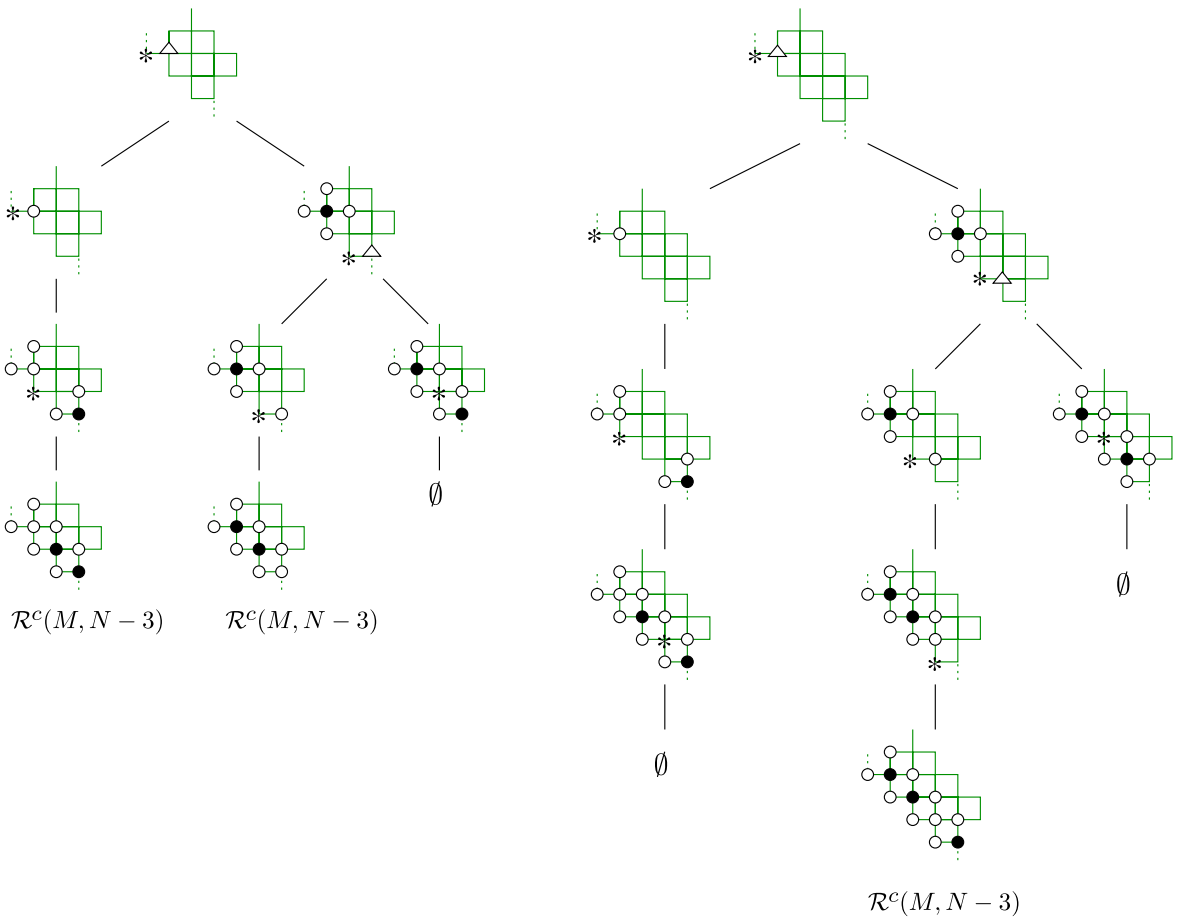

Fig. 8 The top of the matching trees of $\Sigma\left(\mathcal{R}^{c}(6,5)\right)$ and $\Sigma\left(\mathcal{R}^{c}(8,5)\right)$. This figure illustrates what happens for $M \equiv_{3} 0$ and $M \equiv_{3} 2$. We leave it to the reader to practice with the case $M \equiv_{3} 1$

Properties $(A)$ and $(B)$ easily follow by induction on $N$.

\section{Parallelograms with open boundary conditions}

For $K, N \geq 1$, consider now the subgraph $\mathcal{P}(K, N)$ of the square grid induced by the vertices $(x, y)$ satisfying

$$
0 \leq y \leq K-1 \quad \text { and } \quad-y \leq x \leq-y+N-1 .
$$

An example is shown on Figure 9 . We denote by $Z_{\mathcal{P}}(K, N)$ the alternating number of independent sets on the graph $\mathcal{P}(K, N)$.

Theorem 8 Let $K, N \geq 1$. Let $m=\lceil 2 K / 3\rceil$.

- If $K \equiv{ }_{3} 1$, then

- if $N \equiv_{3} 1$ then $\Sigma(\mathcal{P}(K, N))$ is contractible and $Z_{\mathcal{P}}(K, N)=0$,

- otherwise, $\Sigma(\mathcal{P}(K, N))$ is homotopy equivalent to a sphere of dimension mn1 , with $n=\lceil N / 3\rceil$, and $Z_{\mathcal{P}}(K, N)=(-1)^{n}$.

- If $K \equiv_{3} 2$, write $N=2 q K+r$, with $0 \leq r \leq 2 K-1$. 
Fig. 9 The parallelogram graph $\mathcal{P}(4,8)$
$(0,0)$

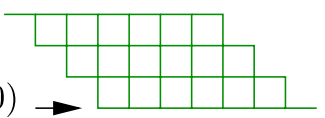

- If $r \equiv_{3} 1,2$, then $\Sigma(\mathcal{P}(K, N))$ is contractible and $Z_{\mathcal{P}}(K, N)=0$,

- otherwise $\Sigma(\mathcal{P}(K, N))$ is homotopy equivalent to a sphere of dimension mn -1 with $n=\left\lceil\frac{2 K-1}{2 K} \cdot \frac{N}{3}\right\rceil$, and $Z_{\mathcal{P}}(K, N)=1$.

- If $K \equiv{ }_{3} 0$, write $N=2 q(K+1)+r$ with $0 \leq r \leq 2 K+1$.

- If $r \equiv_{3} 0$ with $r \geq 1$, or $r \equiv_{3} 1$ with $r \leq 2 K$, then $\Sigma(\mathcal{P}(K, N))$ is contractible and $Z_{\mathcal{P}}(K, N)=0$,

- otherwise, $\Sigma(\mathcal{P}(K, N))$ is homotopy equivalent to a sphere of dimension mn - 1 where $n=\left\lceil\frac{2 K+3}{2 K+2} \cdot \frac{N}{3}\right\rceil$, and $Z_{\mathcal{P}}(K, N)=1$.

Remark We prove in Corollary 12 that for $N \equiv_{3} 1$ and $K$ large enough, the alternating number $Z_{\mathcal{P}}(K, N ; C, D)$ of independent sets on the parallelogram $\mathcal{P}(K, N)$ having prescribed conditions $C$ and $D$ on the top and bottom row is 0 , for all configurations $C$ and $D$. This is not in contradiction with the above theorem: for $K$ large enough, if $K \not \equiv_{3} 1$, the quotient $q$ appearing in the theorem is simply 0 , so that the condition $N \equiv_{3} 1$ boils down to $r \equiv_{3} 1$, with $r$ small compared to $K$, and $Z_{\mathcal{P}}(K, N)=0$.

Proof We construct a Morse matching of the graph $\mathcal{P}(K, N)$ by applying the general method of Section 2. We then show that for all values of $K$ and $N$, the matching thus obtained has at most one unmatched cell, of cardinality $m n$. As before, Corollary 2 completes the proof.

The results in the case $K \equiv_{3} 1$ are reminiscent of what we obtained for rectangles (Theorem 6). The rule for choosing tentative pivots and splitting vertices is the same as before, and the proof follows the same principles. We do not repeat the argument.

The other two congruence classes of $K$ are more complicated and require a different pivot choice. Let us begin with the case $K \equiv_{3} 2$. First, we partition the set of vertices of $\mathcal{P}(K, \infty)$ into triangular subsets $\mathcal{T}_{1}, \mathcal{T}_{2}, \ldots$ defined by:

$$
\begin{aligned}
\mathcal{T}_{2 \ell+1} & =\{(x, y): 0 \leq y<K, 2 \ell K \leq x+y, x-y<2 \ell K+1\}, \\
\mathcal{T}_{2 \ell} & =\{(x, y): 0 \leq y<K, 2(\ell-1) K+1 \leq x-y, x+y<2 \ell K\} .
\end{aligned}
$$

These triangles are shown in Figure 10 for $K=5$.

We now describe the pivot order. The pivots are first taken in $\mathcal{T}_{1}$, then in $\mathcal{T}_{2}$ (once all vertices of $\mathcal{T}_{1}$ are prescribed), and so on. The pivot order for $\mathcal{T}_{1}$ is very similar to what we have done previously. That is, we follow the diagonals of slope -1 from upper left to lower right but with the restriction that we must stay within the triangle. For $\mathcal{T}_{2}$ the pivot order is what we get when turning $\mathcal{T}_{1}$ upside down. That is, we follow the diagonals of slope 1 from bottom left to top right.

Denote by $U^{p}(K, N)$ the set of unmatched elements of $\Sigma(\mathcal{P}(K, N))$. We observe (Figure 10) that, once all pivots in $\mathcal{T}_{1}$ and $\mathcal{T}_{2}$ have been exhausted, only one node $\Sigma(A, B)$ of the matching tree is non-empty. The prescribed vertices of this node are 
Fig. 10 The configuration of prescribed vertices when the pivots of $\mathcal{T}_{1}$ and $\mathcal{T}_{2}$ have been exhausted, for $K=5$. This configuration occurs in the non-contractible branch of the matching tree. The pivots, indicated by $*$ and numbered by pivot order, and the splitting vertices $\Delta$, are empty. Each time a partial matching is performed, some new prescribed vertices appear: they are joined by thick lines

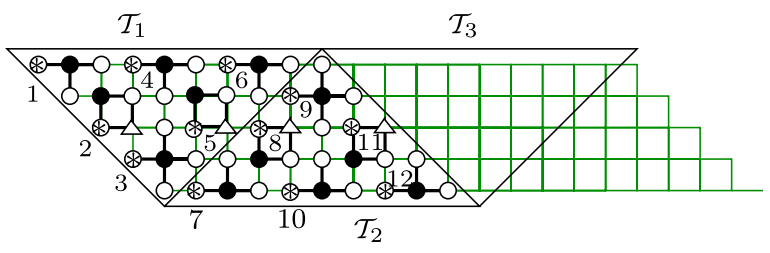

those of $A \cup B=\mathcal{T}_{1} \cup \mathcal{T}_{2}$. This gives, for $N \geq 2 K$ :

$$
U^{p}(K, N) \cong U^{p}(K, 2 K) * U^{p}(K, N-2 K),
$$

which is key to our induction on $N$. By convention, $\mathcal{P}(K, 0)$ is the empty graph, and its unique independent set is the empty set, so that $\left|U^{p}(K, 0)\right|=1$. We also note that the unique unmatched element of $\Sigma(\mathcal{P}(K, 2 K))$ has cardinality $m(m-1)=$ $(2 K+2)(2 K-1) / 9$.

Upon iterating the above identity, we obtain, if $N=2 q K+r$,

$$
U^{p}(K, N) \cong U^{p}(K, 2 q K) * U^{p}(K, r),
$$

where the only unmatched cell of $\Sigma(\mathcal{P}(K, 2 q K))$ has $q m(m-1)$ vertices. It thus remains to describe what our matching rule produces for the graphs $\mathcal{P}(K, r)$, for $0 \leq r \leq 2 K-1$. The following properties are easily observed on the example of Figure 10.

(1) If $r \equiv_{3} 1$, the rightmost vertex in the top row of $\mathcal{P}(K, r)$, which belongs to the triangle $\mathcal{T}_{1}$, becomes a free pivot at some stage of the matching procedure, so that $\left|U^{p}(K, r)\right|=0$.

(2) If $r \equiv_{3} 2$, the rightmost vertex in the top row of $\mathcal{P}(K, r) \cap \mathcal{T}_{2}$ becomes a free pivot at some stage, so that again, $\left|U^{p}(K, r)\right|=0$.

(3) Finally, if $r \equiv{ }_{3} 0$, we obtain a unique unmatched cell, of cardinality $m r / 3$. More precisely, for $0 \leq i<r / 3$, the three diagonals defined by $3 i \leq x+y \leq 3 i+2$, taken together, contain $m$ vertices of the unmatched cell.

Putting together our recursion and the above results for $\mathcal{P}(K, r)$, we find that the only non-contractible cases are when $r \equiv_{3} 0$. In this case, there is only one unmatched element in $\Sigma(\mathcal{P}(K, N))$, of cardinality $q m(m-1)+m r / 3$. The result follows for the case $K \equiv{ }_{3} 2$.

Let us now adapt this to the final case $K \equiv_{3} 0$. The triangles that we used to define the pivot rule now become trapezoids $\mathcal{T}_{1}, \mathcal{T}_{2}, \ldots$, defined by:

$$
\begin{aligned}
\mathcal{T}_{2 \ell+1} & =\{(x, y): 0 \leq y<K, 2 \ell(K+1) \leq x+y, x-y<2 \ell(K+1)+2\}, \\
\mathcal{T}_{2 \ell} & =\{(x, y): 0 \leq y<K, 2(\ell-1)(K+1)+2 \leq x-y, x+y<2 \ell(K+1)\} .
\end{aligned}
$$


Fig. 11 The configuration of prescribed vertices when the pivots of $\mathcal{T}_{1}$ and $\mathcal{T}_{2}$ have been exhausted, for $K=6$

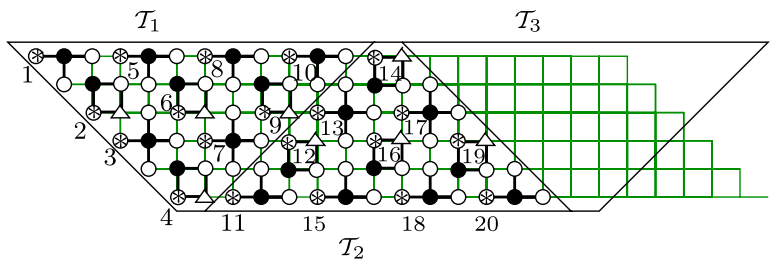

These trapezoids are shown in Figure 11 for $K=6$. The pivots are then chosen using the same rule as in the case $K \equiv_{3} 2$. Again, one observes that when the pivots of $\mathcal{T}_{1}$ and $\mathcal{T}_{2}$ have been exhausted, only one vertex $\Sigma(A, B)$ of the matching tree is nonempty, and its prescribed vertices are those of $\mathcal{T}_{1} \cup \mathcal{T}_{2}$. This gives, for $N \geq 2 K+2$ :

$$
U^{p}(K, N) \cong U^{p}(K, 2 K+2) * U^{p}(K, N-2 K-2) .
$$

Moreover, $\Sigma(\mathcal{P}(K, 2 K+2))$ has a unique unmatched cell, of cardinality $m(m+1)$. Let us write $N=2 q(K+1)+r$, with $0 \leq r \leq 2 K+1$. Iterating the above identity gives

$$
U^{p}(K, N) \cong U^{p}(K, 2 q(K+1)) * U^{p}(K, r),
$$

where the only unmatched cell of $\Sigma(\mathcal{P}(K, 2 q(K+1)))$ has cardinality $q m(m+1)$.

It remains to describe what our matching rule produces for the graphs $\mathcal{P}(K, r)$, for $0 \leq r \leq 2 K+1$. We refer again to Figure 11 .

(1) If $r \equiv_{3} 1$, with $r \leq 2 K$, the rightmost vertex in the top row of $\mathcal{P}(K, r)$ becomes a free pivot at some stage of the procedure, so that $\left|U^{p}(K, r)\right|=0$.

(2) If $r=2 K+1$, there is a unique unmatched cell, with cardinality $m(m+1)$ (it coincides with the unmatched cell obtained for $\mathcal{P}(K, 2 K+2))$.

(3) If $r \equiv{ }_{3} 0$ with $r>0$, the rightmost vertex in the top row of $\mathcal{P}(K, r) \cap \mathcal{T}_{2}$ becomes a free pivot at some stage of the matching procedure, so that again, $\left|U^{p}(K, r)\right|=$ 0 .

(4) If $r=0$, we have the empty graph, with the empty set as unique (and unmatched) independent set.

(5) Finally, if $r \equiv_{3} 2$, we obtain a unique unmatched cell, of cardinality $m(r+1) / 3$.

Putting together our recursion and the above results for $\mathcal{P}(K, r)$, we find that the only non-contractible cases are when $r=0, r=2 K+1$ and $r \equiv{ }_{3} 2$. In these cases, there is only one unmatched element in $\Sigma(\mathcal{P}(K, N))$, of cardinality $q m(m+1)+m\lceil r / 3\rceil$. The result follows for the case $K \equiv{ }_{3} 0$.

Remark The parallelogram $\mathcal{P}(K, N)$ gives rise to two distinct families of shapes with cylindric boundary conditions:

- Gluing the two diagonal borders of $\mathcal{P}(K, N+1)$ by identifying the points $(-i, i)$ and $(-i+N, i)$ for $0 \leq i \leq K-1$ gives the "usual" cylinder $\mathbf{Z} / N \mathbf{Z} \times$ $\{0,1, \ldots, K-1\}$. It is conjectured in [8] that for odd $N$ the corresponding alternating number of independent sets is 1 , except for the case $N \equiv_{6} 3, K \equiv{ }_{3} 1$ when it is conjectured to be -2 . 
- Gluing the two horizontal borders of $\mathcal{P}(K+1, N)$ by identifying the points $(i, 0)$ and $(i-K, K)$ for $0 \leq i \leq N-1$ gives $\mathcal{R}^{c}(2 K, N)$, the cylindric version of the rectangle which we studied in Section 4.

\section{Transfer matrices}

\subsection{Generalities}

We develop here a general (and very classical) transfer matrix framework which we will apply later to the enumeration of independent sets on various subgraphs of the square lattice. See [12, Ch. 4] for generalities on transfer matrices.

Let $r \geq 1$, and let $\mathcal{S}$ be a collection of subsets in $\llbracket 1, r \rrbracket:=\{1,2, \ldots, r\}$, with $\operatorname{card}(\mathcal{S})=d$. Let $\mathbb{T}$ be a square matrix of size $d$, with complex coefficients, whose rows and columns are indexed by the elements of $\mathcal{S}$. The entry of $\mathbb{T}$ lying in row $C$ and column $D$ is denoted $\mathbb{T}(C, D)$. A configuration of length $n$ is any sequence $I=\left(C_{0}, C_{1}, \ldots, C_{n}\right)$ of subsets of $\mathcal{S}$. We say that $C_{0}$ and $C_{n}$ are the borders of $I$. The weight of $I$ is

$$
w(I)=\prod_{i=0}^{n-1} \mathbb{T}\left(C_{i}, C_{i+1}\right)
$$

A cyclic configuration of length $n$ is a configuration $I=\left(C_{0}, C_{1}, \ldots, C_{n-1}, C_{n}\right)$ such that $C_{0}=C_{n}$. Observe that for all $C \in \mathcal{S}, I=(C)$ is a cyclic configuration of length 0 , so that there are exactly $d$ such configurations.

Let $t$ be an indeterminate. It is well-known, and easy to prove (see for instance [12, Thm. 4.7.2]), that the length generating function of configurations with prescribed borders $C$ and $D$, weighted as above, is

$$
G_{C, D}(t):=\sum_{n \geq 0} \sum_{I=\left(C, C_{1}, \ldots, C_{n-1}, D\right)} t^{n} w(I)=(1-t \mathbb{T})^{-1}(C, D) .
$$

From this, one derives that the length generating function of cyclic configurations is the trace of $(1-t \mathbb{T})^{-1}$ :

$$
G^{c}(t):=\sum_{C} G_{C, C}(t)=\operatorname{tr}(1-\mathrm{t} \mathbb{T})^{-1}
$$

In what follows, we will be interested in deriving eigenvalues of the transfer matrix $\mathbb{T}$ from the generating functions $G_{C, D}(t)$ and $G^{c}(t)$. This is motivated by the work of Fendley et al. who conjectured that the eigenvalues of various transfer matrices related to the enumeration of independent sets are roots of unity [3]. One first observation is that finding the whole spectrum $\left(\lambda_{1}, \ldots, \lambda_{d}\right)$ of the transfer matrix is equivalent to finding the generating function of cyclic configurations: indeed,

$$
G^{c}(t)=\operatorname{tr}(1-t \mathbb{T})^{-1}=\sum_{n \geq 0} t^{n} \operatorname{tr}\left(\mathbb{T}^{n}\right)=\sum_{n \geq 0} t^{n}\left(\lambda_{1}^{n}+\cdots+\lambda_{d}^{n}\right)=\sum_{i=1}^{d} \frac{1}{1-\lambda_{i} t}
$$




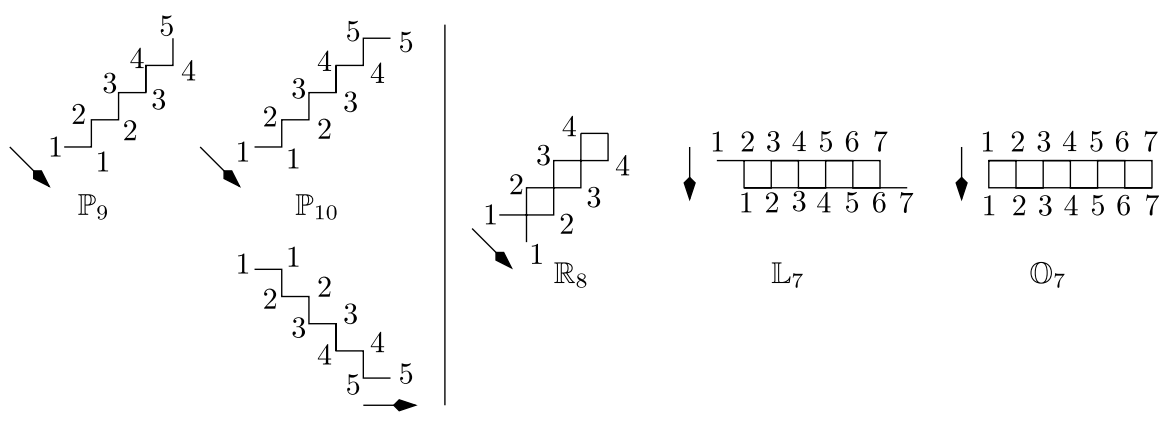

Fig. 12 How the various transfer matrices act

From (3), (4), and the classical formula giving the inverse of a matrix in terms of its determinant and its comatrix, one concludes that

- For every pair $C, D$, the reciprocals of the poles of $G_{C, D}(t)$ are eigenvalues of $\mathbb{T}$,

- Conversely, the set of non-zero eigenvalues of $\mathbb{T}$ coincides with the set of reciprocals of poles of the series $G_{C, C}(t)$, for $C$ running over $\mathcal{S}$.

In other words: counting configurations with cyclic boundary conditions gives the whole spectrum; at least partial information can be derived from the enumeration of configurations with open boundary conditions.

This general framework will be specialized below to the case where the sets $C$ and $D$ describe hard-particle configurations (a.k.a. independent sets) on certain layers on the square lattice. The weights $\mathbb{T}(C, D)$ will be designed in such a way that the weight of a configuration $I$ is 0 if $I$ is not an independent set, and $(-1)^{|I|}$ otherwise. We have schematized in Figure 12 the various transfer matrices we consider. They will be defined precisely in the text.

\subsection{Two complete spectra}

In this section, we combine the above generalities with the results obtained in Section 4 for the alternating number of independent sets on the cylinders $\mathcal{R}^{c}(M, N)$. As these cylinders can be generated from two types of transfer matrices (called $\mathbb{R}_{N}$ and $\mathbb{L}_{N}$ in Figure 12), we obtain the complete spectra of these two families of transfer matrices. All the non-zero eigenvalues are found to be roots of unity.

We begin with a transfer matrix $\mathbb{P}_{N}$ that describes the independent sets of the 2-diagonal graph $\mathcal{R}(2, N)$. The rows of $\mathbb{P}_{N}$ are indexed by subsets $C$ of $\{1,2, \ldots,\lceil N / 2\rceil\}$, its columns are indexed by subsets $D$ of $\{1,2, \ldots,\lfloor N / 2\rfloor\}$, and

$$
\mathbb{P}_{N}(C, D)= \begin{cases}i^{|C|+|D|}, & \text { if } C \cap D=C \cap(D+1)=\emptyset, \\ 0, & \text { otherwise. }\end{cases}
$$


The notation $D+1$ means $\{i+1: i \in D\}$. Observe that $\mathbb{P}_{N}$ is not a square matrix if $N$ is odd. ${ }^{1}$ However, if $\overline{\mathbb{P}}_{N}$ denotes the transpose of $\mathbb{P}_{N}$, then $\mathbb{R}_{N}:=\mathbb{P}_{N} \overline{\mathbb{P}}_{N}$ is the (square) transfer matrix corresponding to the graph $\mathcal{R}(3, N)$ : for $C, D \subseteq$ $\{1, \ldots,\lceil N / 2\rceil\}$,

$$
\mathbb{R}_{N}(C, D)=\sum_{E \subseteq\{1, \ldots,\lfloor N / 2\rfloor\}} \mathbb{P}_{N}(C, E) \overline{\mathbb{P}}_{N}(E, D)=i^{|C|+|D|} \sum_{E: I=(C, E, D) \text { ind.set }}(-1)^{|E|}
$$

is, up to the factor $i^{|C|+|D|}$, the alternating number of independent sets $I$ of $\mathcal{R}(3, N)$, with top and bottom borders $C$ and $D$ respectively (the set $E$ describes the elements of $I$ lying on the central diagonal). The coefficients of this matrix actually have a simpler expression. Indeed, the configuration $(C, E, D)$ is an independent set if and only if $E$ is in the complement of $C \cup D \cup(C-1) \cup(D-1)$. Hence the sum of terms $(-1)^{|E|}$ is 0 unless this complement is empty. That is, for $C, D \subseteq\{1,2, \ldots,\lceil N / 2\rceil\}$,

$$
\mathbb{R}_{N}(C, D)= \begin{cases}i^{|C|+|D|}, & \text { if }\{1,2, \ldots,\lfloor N / 2\rfloor\}=C \cup D \cup(C-1) \cup(D-1), \\ 0, & \text { otherwise. }\end{cases}
$$

From (5), one derives that the entry $(C, C)$ in the product $\mathbb{R}_{N}^{k}=\left(\mathbb{P}_{N} \overline{\mathbb{P}}_{N}\right)^{k}$ is the alternating number of independent sets on the cylinder $\mathcal{R}^{c}(2 k, N)$ studied in Section 4, with border condition $C$ on the first diagonal. By Section 6.1, these numbers are related to the spectrum $\left(\lambda_{1}, \ldots, \lambda_{d}\right)$ of the transfer matrix $\mathbb{R}_{N}$. More precisely, (4) gives:

$$
G^{c}(t)=d+\sum_{k \geq 1} Z_{\mathcal{R}}^{c}(2 k, N) t^{k}=\sum_{i=1}^{d} \frac{1}{1-\lambda_{i} t}
$$

where the numbers $Z_{\mathcal{R}}^{c}(2 k, N)$ are given in Theorem 7 and $d=2^{\lceil N / 2\rceil}$.

Theorem 9 Let $N \geq 1$. The transfer matrix $\mathbb{R}_{N}$ defined by (6) has size $2^{\lceil N / 2\rceil}$. If $N \equiv \equiv_{3} 1$, then $\mathbb{R}_{N}$ is nilpotent (all its eigenvalues are 0 ). Otherwise, $\mathbb{R}_{N}$ has eigenvalues:

- 0 with multiplicity $2^{\lceil N / 2\rceil}-2^{n}$,

- 1 with multiplicity $\left(2^{n}+2(-1)^{n}\right) / 3$,

- $j$ and $j^{2}$ with multiplicity $\left(2^{n}-(-1)^{n}\right) / 3$,

where $j=e^{2 i \pi / 3}$ and $n=\lceil N / 3\rceil$.

Proof We start from the identity

$$
G^{c}(t)=d+\sum_{k \geq 1} Z_{\mathcal{R}}^{c}(2 k, N) t^{k}=\operatorname{tr}\left(1-t \mathbb{R}_{N}\right)^{-1}=\sum_{i=1}^{d} \frac{1}{1-\lambda_{i} t}
$$

\footnotetext{
${ }^{1}$ When $N$ is even, the matrix $\mathbb{P}_{N}$ also describes the independent sets of the parallelogram $\mathcal{P}(N / 2,2)$, as illustrated in Figure 12. Accordingly, its powers will be used later to count independent sets on the parallelograms $\mathcal{P}(N / 2, \cdot)$.
} 
This allows us to read off the eigenvalues directly from the generating function of the numbers $Z_{\mathcal{R}}^{c}(2 k, N)$, given by Theorem 7 .

When $N \equiv{ }_{3} 1, G^{c}(t)=d$ and so all the eigenvalues of $\mathbb{R}_{N}$ are zero. Otherwise,

$$
G^{c}(t)=d+2^{n} \frac{t^{3}}{1-t^{3}}+(-1)^{n} \frac{t+t^{2}}{1-t^{3}},
$$

and the result follows by a partial fraction expansion.

In the case $N \equiv_{3} 1$, the above theorem gives an unexpected strengthening of Theorem 6. This observation was communicated to us by Alan Sokal, merci à lui !

Corollary 10 Let $N \equiv_{3} 1$. For $M>2^{1+\lceil N / 2\rceil}$, the alternating number $Z_{\mathcal{R}}(M, N$; $C, D)$ of independent sets on the rectangle $\mathcal{R}(M, N)$ having prescribed conditions $C$ and $D$ on the two extreme diagonals of slope 1 is 0 , for all configurations $C$ and $D$.

Proof First assume that $M=2 m+1$, so that $m \geq 2^{\lceil N / 2\rceil}$. By the above theorem and the Cayley-Hamilton theorem, the $m$ th power of $\mathbb{R}_{N}$ vanishes. Thus by (3), the series $G_{C, D}(t)$ is a polynomial in $t$ of degree at most $2^{\lceil N / 2\rceil}-1$. But the coefficient of $t^{m}$ in this series is precisely $Z_{\mathcal{R}}(2 m+1, N ; C, D)$.

Similarly, if $M=2 m+2$ with $m \geq 2^{\lceil N / 2\rceil}$, the number $Z_{\mathcal{R}}(M, N ; C, D)$ is the entry $(C, D)$ in the matrix $\mathbb{R}_{N}^{m} \mathbb{P}_{N}$, which vanishes.

As observed at the end of Section 5, the cylindric shape $\mathcal{R}^{c}(2 k, N)$ can also be obtained by wrapping the parallelogram $\mathcal{P}(k+1, N)$ on a cylinder, identifying the top and bottom (horizontal) layers. Consequently, the results of Theorem 7 also give the spectrum of another transfer matrix, denoted $\mathbb{L}_{N}$, which describes how to construct the shapes $\mathcal{P}(\cdot, N)$ layer by layer (Figure 12). The rows and columns of $\mathbb{L}_{N}$ are indexed by independent sets of the segment $\mathcal{P}(1, N)$. Thus the size of $\mathbb{L}_{N}$ is the Fibonacci number $F_{N+1}$, with $F_{0}=F_{1}=1$ and $F_{N+1}=F_{N}+F_{N-1}$, and if $C$ and $D$ are independent sets of $\mathcal{P}(1, N)$,

$$
\mathbb{L}_{N}(C, D)= \begin{cases}i^{|C|+|D|}, & \text { if } C \cap(D+1)=\emptyset, \\ 0, & \text { otherwise. }\end{cases}
$$

The generalities of Section 6.1 imply that the spectrum $\left(\mu_{1}, \ldots, \mu_{d}\right)$ of $\mathbb{L}_{N}$ satisfies

$$
F_{N+1}+\sum_{k \geq 1} Z_{\mathcal{R}}^{c}(2 k, N) t^{k}=\sum_{i=1}^{F_{N+1}} \frac{1}{1-\mu_{i} t} .
$$

Comparing with (7) shows that the spectra of $\mathbb{L}_{N}$ and $\mathbb{R}_{N}$ coincide, apart from the multiplicity of the null eigenvalue.

Theorem 11 Let $N \geq 1$. The transfer matrix $\mathbb{L}_{N}$ defined by (8) has size $F_{N+1}$. If $N \equiv{ }_{3} 1$, then $\mathbb{L}_{N}$ is nilpotent. Otherwise, $\mathbb{L}_{N}$ has eigenvalues:

- 0 with multiplicity $F_{N+1}-2^{n}$, 
- 1 with multiplicity $\left(2^{n}+2(-1)^{n}\right) / 3$,

- $j$ and $j^{2}$ with multiplicity $\left(2^{n}-(-1)^{n}\right) / 3$,

where $j=e^{2 i \pi / 3}$ and $n=\lceil N / 3\rceil$.

As for the matrix $\mathbb{R}_{N}$, the nilpotent case $N \equiv_{3} 1$ gives the following corollary, which has to be compared to Theorem 8 .

Corollary 12 Let $N \equiv_{3} 1$. Then, for $K>F_{N+1}$, the alternating number $Z_{\mathcal{P}}(K, N$; $C, D)$ of independent sets on the parallelogram $\mathcal{P}(K, N)$ having prescribed conditions $C$ and $D$ on the top and bottom row is 0 , for all configurations $C$ and $D$.

Proof The number $Z_{\mathcal{P}}(K, N ; C, D)$ is the entry $(C, D)$ in the $(K-1)$ th power of the transfer matrix $\mathbb{L}_{N}$. But this power vanishes by the Cayley-Hamilton theorem.

\subsection{Partial results on two other spectra}

In this section we focus on two transfer matrices that generate the usual cylinder $\mathcal{C}(K, N):=\{0,1, \ldots, K-1\} \times \mathbf{Z} / N \mathbf{Z}$. This cylinder can be obtained by identifying the diagonal borders of the parallelogram $\mathcal{P}(K, N+1)$. Alternatively, it can be obtained by wrapping the ordinary $K \times(N+1)$ rectangle $\{0,1, \ldots, K-1\} \times$ $\{0,1, \ldots, N\}$ on a cylinder, identifying the vertices $(i, 0)$ and $(i, N)$. Underlying the first construction are the matrices $\mathbb{P}_{2 K}$ defined at the beginning of Section 6.2 (see (5) and Figure 12). Underlying the second construction is the transfer matrix $\mathbb{O}_{K}$ that describes how to construct the ordinary rectangles of width $K$. This matrix has size $F_{K+1}$, the $(K+1)$ st Fibonacci number, and its rows and columns are indexed by independent sets of the $K$ point segment. If $C$ and $D$ are two of these independent sets,

$$
\mathbb{O}_{K}(C, D)= \begin{cases}i^{|C|+|D|}, & \text { if } C \cap D=\emptyset, \\ 0, & \text { otherwise. }\end{cases}
$$

The similarity with the definition (8) of the matrix $\mathbb{L}_{N}$ is striking, but the spectrum of $\mathbb{O}_{K}$ is definitely more complex than that of $\mathbb{L}_{N}$. It is conjectured in [3] that all the eigenvalues of $\mathbb{O}_{K}$ are roots of unity.

Let $Z_{\mathcal{C}}(K, N)$ denote the alternating number of independent sets on the cylinder $\mathcal{C}(K, N)$. From the generalities of Section 6.1, we have:

$$
\sum_{N \geq 1} Z_{\mathcal{C}}(K, N) t^{N}=\operatorname{tr}\left(1-t \mathbb{P}_{2 K}\right)^{-1}-2^{K}=\operatorname{tr}\left(1-t \mathbb{O}_{K}\right)^{-1}-F_{K+1}
$$

That is, the spectra of the matrices $\mathbb{P}_{2 K}$ and $\mathbb{O}_{K}$ coincide, apart from the multiplicity of the null eigenvalue.

Alas, we do not know what the numbers $Z_{\mathcal{C}}(K, N)$ are. However, recall from Section 6.1 that enumerative results on configurations with open boundary conditions provide partial information on the spectrum of the transfer matrix. Here, we exploit the results of Section 5 on parallelograms to obtain some information on the spectrum of $\mathbb{P}_{2 K}$ (and thus of $\mathbb{O}_{K}$ ). 
For all $C, D \subseteq\{1, \ldots, K\}$, let $Z_{\mathcal{P}}(K, N ; C, D)$ be the alternating number of independent sets of the parallelogram $\mathcal{P}(K, N)$ having borders $C$ and $D$ respectively on the leftmost and rightmost diagonal. Then (3) gives:

$$
\begin{aligned}
G_{C, D}(t) & =\left(1-t \mathbb{P}_{2 K}\right)^{-1}(C, D) \\
& =\delta_{C, D}+(-i)^{|C|+|D|} \sum_{I=\left(C, C_{1}, \ldots, C_{n-1}, D\right), n \geq 1}(-1)^{|I|} t^{n} \\
& =\delta_{C, D}+(-i)^{|C|+|D|} \sum_{N \geq 1} Z_{\mathcal{P}}(K, N+1 ; C, D) t^{N} .
\end{aligned}
$$

Note that in the first formula, the weight $(-i)^{|C|+|D|}(-1)^{|I|}$ results in a weight $i$ for each vertex of the extreme diagonals, as it should. Since the numbers $Z_{\mathcal{P}}(K, N+$ $2 ; \emptyset, \emptyset)$ coincide with the numbers $Z_{\mathcal{P}}(K, N)$ given in Theorem 8 , this allows us to find some eigenvalues of $\mathbb{P}_{2 K}$ and $\mathbb{O}_{K}$. We indicate in the next section how our pivot principle can be extended to count independent sets with prescribed borders, so as to determine more series $G_{C, D}$ and thus more eigenvalues of the matrix $\mathbb{O}_{K}$.

Proposition 13 The transfer matrix $\mathbb{O}_{K}$ defined by (9) satisfies the following properties.

- If $K \equiv_{3} 1$, then $e^{i \pi / 3}$ and $e^{-i \pi / 3}$ are eigenvalues of $\mathbb{O}_{K}$.

- If $K \equiv_{3} 2$, then all the $2 K$ th roots of unity, except maybe -1 , are eigenvalues of $\mathbb{O}_{K}$.

- If $K \equiv_{3} 0$, then all the $(2 K+2)$ th roots of unity, except maybe -1 and, if $K$ is odd, $\pm i$, are eigenvalues of $\mathbb{O}_{K}$.

Proof Specializing (10) to $C=D=\emptyset$ gives

$$
G_{\emptyset, \emptyset}(t)=\left(1-t \mathbb{P}_{2 K}\right)^{-1}(\emptyset, \emptyset)=1+\sum_{N \geq 0} Z_{\mathcal{P}}(K, N) t^{N+1},
$$

with $Z_{\mathcal{P}}(K, 0)=1$. Recall that the reciprocals of the poles of this series are eigenvalues of $\mathbb{O}_{K}$ and $\mathbb{P}_{2 K}$. The numbers $Z_{\mathcal{P}}(K, N)$ are given in Theorem 8 . If $K \equiv_{3} 1$,

$$
G_{\emptyset, \emptyset}=1+t+\sum_{n \geq 1}(-1)^{n}\left(t^{3 n}+t^{3 n+1}\right)=\frac{1}{1-t+t^{2}} .
$$

If $K \equiv{ }_{3} 2$,

$$
G_{\emptyset, \emptyset}=1+\sum_{q \geq 0} \sum_{p=0}^{(2 K-1) / 3} t^{2 q K+3 p+1}=1+\frac{t}{1-t^{2 K}} \frac{1-t^{2 K+2}}{1-t^{3}} .
$$

Note that $\left(1-t^{2 K+2}\right) /\left(1-t^{3}\right)$ is a polynomial, and that the only root this polynomial shares with $1-t^{2 K}$ is -1 . 
Finally, if $K \equiv_{3} 0$,

$$
\begin{aligned}
G_{\emptyset, \emptyset} & =1+\sum_{q \geq 0} t^{2 q(K+1)+1}+\sum_{q \geq 0} t^{2 q(K+1)+2 K+2}+\sum_{q \geq 0} \sum_{p=0}^{2 K / 3-1} t^{2 q(K+1)+3 p+3} \\
& =\frac{1}{1-t^{2 K+2}}\left(1+t+t^{3} \frac{1-t^{2 K}}{1-t^{3}}\right) .
\end{aligned}
$$

Note that $\left(1+t+t^{3}\left(1-t^{2 K}\right) /\left(1-t^{3}\right)\right)$ is a polynomial. The roots it shares with $\left(1-t^{2 K+2}\right)$ are -1 , and, if $K$ is odd, $\pm i$.

Note Even when $K \not \equiv_{3} 1$, we have only determined a linear number of eigenvalues of $\mathbb{O}_{K}$, while the size of this matrix is exponential in $K$. See Section 7.2 for details on the (large) portion of the spectrum of $\mathbb{O}_{K}$ that remains to be explained.

\section{Final comments and perspectives}

\subsection{Other quadrangles}

A natural generalization of the rectangles and parallelograms studied in Sections 3 and 5 is the subgraph $\mathcal{G}(M, N)$ of the square lattice induced by the points $(x, y)$ satisfying

$$
a y \leq x \leq a y+M-1 \text { and } \quad-b x \leq y \leq-b x+N-1 \text {, }
$$

for given values of $a$ and $b$. We have solved above the cases $(a, b)=(1,1)$ and $(a, b)=(-1,0)$. In particular, we have proved that in both cases, the alternating number of independent sets is always 0 or \pm 1 . What about other values of $a$ and $b$ ? The case $(a, b)=(0,0)$, which describes ordinary rectangles, shows that the simplicity of our results cannot be extended to all pairs $(a, b)$. Indeed, even though the eigenvalues of the transfer matrix $\mathbb{O}_{K}$ are conjectured to be roots of unity, the alternating number $Z(K, N)$ of independent sets on a $K \times N$ rectangle does not show any obvious pattern. For instance, for $K=4$,

$$
\begin{aligned}
\sum_{N \geq 0} Z(4, N) t^{N}= & \frac{1+t^{4}}{\left(1-t^{2}\right)\left(1+t^{3}\right)} \\
= & 1+t^{2}-t^{3}+2 t^{4}-t^{5}+3 t^{6}-2 t^{7}+3 t^{8}-3 t^{9}+4 t^{10} \\
& +O\left(t^{11}\right)
\end{aligned}
$$

In particular, $Z(4, N) \sim(-1)^{N} N / 3$ as $N$ goes to infinity.

In contrast, recall that it is conjectured that for an ordinary rectangle with cyclic boundary conditions, the alternating number $Z_{\mathcal{C}}(K, N)$ is 1 or -2 when $N$ is odd [8].

Still, the simplicity of the results obtained for rectangles and parallelograms extends to other quadrangles. For instance, if $a=b=2$, a pivot rule similar to the one 
Fig. 13 The graph $\mathcal{G}(14,17)$

obtained for $a=b=2$
$(0,0)$

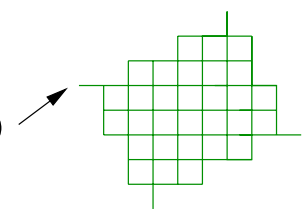

used in the proof of Theorem 6 (the tentative pivot lies as high as possible on the leftmost line of slope -2 ) produces the following results, where the alternating number of independent sets is denoted $Z_{\mathcal{G}}(M, N)$. The proof is left to the reader.

Theorem 14 Let $M, N \geq 1$. Let $m=\lceil M / 5\rceil$ and $n=\lceil N / 5\rceil$.

- If $M \equiv_{5} 0$ and $N \neq 3$, or if $M \equiv_{5} 1$, or if $N \equiv_{5} 1,2$, then $\Sigma(\mathcal{G}(M, N))$ is contractible and $Z_{\mathcal{G}}(M, N)=0$.

- Otherwise, $\Sigma(\mathcal{G}(M, N))$ is homotopy equivalent to a sphere of dimension mn - 1 , and $Z_{\mathcal{G}}(M, N)=(-1)^{m n}$.

It would be worth investigating which values of $a$ and $b$ produce similar results.

\subsection{Transfer matrices}

We have determined in Proposition 13 some of the eigenvalues of the "hard" transfer matrix $\mathbb{O}_{K}$. Recall that all its eigenvalues are conjectured to be roots of unity. We give below the value of the characteristic polynomial $P(K)$ of the matrix $\mathbb{O}(K)$, for $1 \leq K \leq 10$, and split this polynomial into the part that is explained by Proposition 13, and the (bigger and bigger) part that is left unexplained. These data have been obtained with the help of Maple.

$$
\begin{aligned}
& P(1)=\frac{1+t^{3}}{1+t} \cdot 1, \\
& P(2)=\frac{1-t^{4}}{1+t} \cdot 1, \\
& P(3)=\frac{1-t^{8}}{(1+t)\left(1+t^{2}\right)} \cdot 1, \\
& P(4)=\frac{1+t^{3}}{1+t} \cdot\left(1-t^{2}\right)\left(1-t^{4}\right), \\
& P(5)=\frac{1-t^{10}}{1+t} \cdot\left(1+t^{4}\right), \\
& P(6)=\frac{1-t^{14}}{1+t} \cdot\left(1-t^{4}\right)^{2}, \\
& P(7)=\frac{1+t^{3}}{1+t} \cdot \frac{\left(1+t^{4}\right)\left(1-t^{12}\right)\left(1-t^{18}\right)}{1+t^{2}},
\end{aligned}
$$




$$
\begin{aligned}
P(8) & =\frac{1-t^{16}}{1+t} \cdot\left(1-t^{2}\right)\left(1-t^{4}\right)^{2}\left(1+t^{8}\right)\left(1-t^{22}\right) \\
P(9) & =\frac{1-t^{20}}{(1+t)\left(1+t^{2}\right)} \cdot \frac{\left(1+t^{4}\right)\left(1-t^{14}\right)\left(1+t^{10}\right)\left(1-t^{20}\right)\left(1-t^{26}\right)}{1-t^{2}} \\
P(10) & =\frac{1+t^{3}}{1+t} \cdot \frac{\left(1-t^{4}\right)^{2}\left(1-t^{18}\right)^{2}\left(1-t^{24}\right)^{3}\left(1-t^{30}\right)}{1+t^{4}}
\end{aligned}
$$

By Section 6.1, we know that each missing factor occurs in at least one of the series $G_{C, C}(t)$ counting independent sets of the parallelogram $\mathcal{P}(K, \cdot)$ with prescribed border $C$ on extreme diagonals. Conversely, any series $G_{C, D}(t)$ may provide some of these missing factors (see (3)). Hence the following question: can our pivot approach be recycled to compute some of these series, and do we obtain new eigenvalues in this way?

For $C, D \subseteq \llbracket 1, K \rrbracket$, denote by $Z_{\mathcal{P}}(K, N ; C, D)$ the alternating number of independent sets of $\mathcal{P}(K, N)$ having border conditions $C$ and $D$, respectively, on the first (last) diagonal. (This notation was already introduced in Section 6.3.) Recall in particular the connection (10) between these numbers and the series $G_{C, D}(t)$.

Take $K=4$ and $C=\{2,3\}$. If the configuration is $C$ on the first diagonal, then, in the second diagonal, only the vertex labeled 4 may belong to an independent set. This gives

$$
\begin{aligned}
Z_{\mathcal{P}}(4, N ;\{2,3\}, D) & =Z_{\mathcal{P}}(4, N-1 ; \emptyset, D)+Z_{\mathcal{P}}(4, N-1 ;\{4\}, D) \\
& =Z_{\mathcal{P}}(4, N-1 ; \emptyset, D)+Z_{\mathcal{P}}(4, N-4 ;\{2,3\}, D) .
\end{aligned}
$$

The second identity is obtained by applying the pivot rule of Section 3 to the independent sets counted by $Z_{\mathcal{P}}(4, N-1 ;\{4\}, D)$ (Figure 14). The above identity is valid for $N \geq 7$. We first specialize it to $D=\emptyset$, and work out what happens for small values of $N$. Upon summing over $N$, we obtain

$$
G_{C, \emptyset}(t)=-\frac{t}{1-t^{4}}\left(G_{\emptyset, \emptyset}(t)-t\right)=-\frac{t}{(1+t)\left(1-t+t^{2}\right)} .
$$

(We have used (11) for the value of $G_{\emptyset, \emptyset .)}$ We then specialize (12) to $D=C=\{2,3\}$. After working out what happens for small values of $N$, we obtain

$$
G_{C, C}(t)=\frac{1-t G_{\emptyset, C}(t)}{1-t^{4}}=\frac{1+t^{2}+t^{3}}{(1+t)\left(1-t^{4}\right)\left(1-t+t^{2}\right)},
$$

which now explains the missing factors $(1+t)\left(1-t^{4}\right)$ in $P(4)$.

Fig. 14 The pivot rule applied to $\mathcal{P}(4, N)$, with first diagonal $\{2,3\}$ and second diagonal $\{4\}$

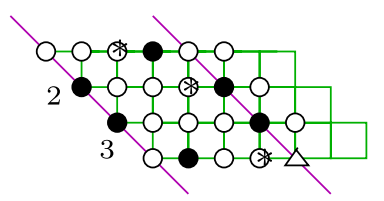


For $K=5$, we obtain similarly the missing factor $1+t^{4}$ by considering the numbers $Z_{\mathcal{P}}(K, N ; C, D)$, with $C=\{3,4\}$. Indeed, after a discussion about the vertex labeled 5 in the second diagonal and a few applications of the pivot rule, one finds

$$
Z_{\mathcal{P}}(K, N ;\{3,4\}, D)=Z_{\mathcal{P}}(K, N-4 ; \emptyset, D)-Z_{\mathcal{P}}(K, N-4 ;\{3,4\}, D),
$$

from which we derive

$$
G_{C, C}(t)=\frac{1}{\left(1-t^{5}\right)\left(1+t^{4}\right)} .
$$

This gives the missing factor $\left(1+t^{4}\right)$.

It would be interesting to know how far one can go with this approach. That is, can we determine all series $G_{C, C}$ in this way? This would allow us to count independent sets on the ordinary cylinder, and thus to find all eigenvalues of the matrix $\mathbb{O}_{K}$.

Note that the largest cyclotomic factor that occurs in the polynomial $P(K)$ seems to be $\Phi_{4 K-10}$.

Remark added to the paper (07 March, 2007): Sonja Cukic and Alexander Engström recently pointed out to us that the following lemma [2, Lemma 2.4] can be used to derive some of our topological results.

Lemma 15 Let $v, w$ be vertices in a graph $G$. If $N(v) \subseteq N(w)$ then $\Sigma(G)$ collapses onto $\Sigma(G-w)$.

Indeed, one can match all independent sets containing $w$ by adding or removing the vertex $v$.

For example, for the rectangles of Theorem 6, one can eliminate the vertices in every third diagonal by a repeated application of Lemma 15. This leaves a graph formed of several paths. One can then eliminate every third vertex in each of these paths. The remaining graph is a disjoint union of edges, and, if $M \equiv_{3} 1$ or $N \equiv_{3} 1$, also isolated vertices. Thus $\Sigma(\mathcal{R}(M, N))$ collapses onto an octahedral sphere (an $m n$-fold join of two points) or to the join of an octahedral sphere with a simplex, respectively. Theorem 6 follows.

Acknowledgements We are grateful to Anders Björner and Richard Stanley for inviting us to the "Algebraic Combinatorics" program at the Institut Mittag-Leffler in Spring 2005, during which part of this work was done. All authors were partially supported by the European Commission's IHRP Programme, grant HPRN-CT-2001-00272, "Algebraic Combinatorics in Europe".

\section{References}

1. Baxter, R.J.: Hard hexagons: exact solution. J. Phys. A 13(3), L61-L70 (1980)

2. Engström, A.: Independence complexes of claw-free graphs. ArXiv:math.CO/0512420 (2005)

3. Fendley, P., Schoutens, K., van Eerten, H.: Hard squares with negative activity. J. Phys. A 38(2), 315-322 (2005), ArXiv:cond-mat/0408497

4. Forman, R.: Morse theory for cell complexes. Adv. Math. 134(1), 90-145 (1998) 
5. Forman, R.: A user's guide to discrete Morse theory. Sém. Lothar. Comb. 48:Art. B48c (electronic) (2002)

6. Jonsson, J.: Simplicial complexes of graphs. PhD thesis, KTH, Stockholm, 2005, to appear in Lecture Notes Math., Springer. http://www.math.kth.se/ jakobj/thesis.html

7. Jonsson, J.: Hard squares on grids with diagonal boundary conditions. Preprint (2006)

8. Jonsson, J.: Hard squares with negative activity and rhombus tilings of the plane. Preprint (2006)

9. Kozlov, D.N.: Complexes of directed trees. J. Comb. Theory Ser. A 88(1), 112-122 (1999)

10. Munkres, J.R.: Elements of Algebraic Topology. Addison-Wesley, Menlo Park (1984)

11. Sloane, N.J.A., Plouffe, S.: The Encyclopedia of Integer Sequences. Academic Press, San Diego (1995). http://www.research.att.com/ njas/sequences/index.html

12. Stanley, R.P.: Enumerative Combinatorics, vol. 1. Cambridge Studies in Advanced Mathematics, vol. 49. Cambridge University Press, Cambridge (1997) 